\title{
Rosiglitazone produces insulin sensitisation by increasing expression of the insulin receptor and its tyrosine kinase activity in brown adipocytes
}

\author{
R. Hernandez, T. Teruel, M. Lorenzo \\ Department of Biochemistry and Molecular Biology II, Faculty of Pharmacy, Complutense University, Madrid, Spain
}

\begin{abstract}
Aims/hypothesis. Rosiglitazone is used to treat Type 2 diabetes because it improves insulin sensitivity. However, the specific molecular mechanism by which this compound acts has not yet been explained.

Methods. We used fetal rat primary brown adipocytes cultured for $24 \mathrm{~h}$ with or without $10 \mu \mathrm{mol} / \mathrm{l}$ rosiglitazone and further stimulated for 5 min with $10 \mathrm{nmol} / 1$ insulin. Next we measured glucose uptake and GLUT4 translocation and submitted the cells to lysis, immunoprecipitation and immunoblotting in order to measure the insulin signalling cascade.

Results. Rosiglitazone noticeably activated basal glucose uptake in a manner dependent on p38-mitogenactivated protein kinase. Rosiglitazone also produced a $40 \%$ increase in insulin-stimulated glucose uptake as a result of increased GLUT4 translocation to the plasma membrane. This happened without changes in the expression of GLUT4 at the mRNA or protein level. This effect correlated with the potentiation by rosiglitazone of insulin-stimulated Tyr phosphorylation of
\end{abstract}

insulin receptor substrate- 1 and to a greater extent of insulin receptor substrate-2. It also correlated with the subsequent activation of phosphatidylinositol 3-kinase and Akt, without changes in protein kinase $\mathrm{C} \zeta$ activity. Rosiglitazone treatment increased insulin receptor expression and insulin-stimulated Tyr phosphorylation of insulin receptor beta-chain, but decreased insulinstimulated Ser phosphorylation. It also potentiated insulin-induced Tyr phosphorylation of insulin receptor beta-chain and protein tyrosine phosphatase $1 \mathrm{~B}$ in coimmunoprecipitates and impaired insulin activation of protein tyrosine phosphatase $1 \mathrm{~B}$ activity.

Conclusions/interpretation. At the insulin receptor level, rosiglitazone-induced improvements of insulin sensitivity result from two convergent mechanisms: increased insulin receptor expression and insulin receptor activation. [Diabetologia (2003) 46:1618-1628]

Keywords Glucose uptake, GLUT4 translocation, insulin receptor, protein tyrosine phosphatase $1 \mathrm{~B}$, Akt, insulin receptor substrate-2, brown adipocytes, rosiglitazone, insulin.
Received: 3 June 2003 / Revised: 24 July 2003

Published online: 1 November 2003

(C) Springer-Verlag 2003

Corresponding author: M. Lorenzo, Department of Biochemistry and Molecular Biology II, Faculty of Pharmacy, Complutense University, 28040 Madrid, Spain

E-mail:mlorenzo@farm.ucm.es

Abbreviations: TZDs, Thiazolidinediones; IR, insulin receptor; PPAR, peroxisome proliferating activated receptor; PI, phosphatidylinositol; MAPK, mitogen-activated protein kinase; PKC, protein kinase C; PTP, protein tyrosine phosphatase; FAS, fatty acid synthase; UCP, uncoupling protein; MBP, myelin basic protein; MEM, minimal essential medium.
Thiazolidinediones (TZDs) like troglitazone, pioglitazone and rosiglitazone are insulin-sensitising drugs that improve insulin action in vivo across a wide spectrum of insulin-resistant states and have recently been introduced for the treatment of Type 2 diabetes. They are agonists for peroxisome proliferating activated receptor (PPAR) $\gamma$ and, although there is increasing evidence that TZDs have action mechanisms which are independent of PPAR $\gamma$, the relative ability of different TZDs to bind and activate PPAR $\gamma$ in vitro correlates with the antidiabetic action of these drugs in rodents $[1,2]$. However, it is far from clear what mechanisms 
are used for TZDs to increase insulin sensitivity. Troglitazone has been shown to improve glucose disposal by insulin in human skeletal muscle and adipose tissue from prediabetic and Type 2 diabetic patients. It also increased Akt phosphorylation, although insulin receptor (IR) kinase and phosphatidylinositol (PI) 3-kinase activities were not changed [3, 4]. Rosiglitazone reduced circulating concentrations of non-esterified fatty acids in Zucker obese rats and potentiated insulin-stimulated Akt phosphorylation in adipose and muscle tissues, but in this case also increased Tyr phosphorylation of IR and IRS-1 [5]. Studies in cellular models have shown that TZDs exert their insulinsensitising effects at the level of PI3-kinase and Akt activation, as the Akt 2 gene seems to be essential for the maintenance of normal glucose homeostasis [6]. However, other evidence suggests that TZDs affect glucose uptake by modulating changes in the expression of IRS-2, p85-PI3-kinase, Cbl-associated protein, GLUT4 or GLUT1, either in adipocyte or muscle cells $[4,7,8,9,10]$.

Brown adipose tissue is a target tissue for insulin action, especially during late fetal development when insulin supports survival and promotes adipogenic and thermogenic differentiation of primary cells $[11,12$, 13]. Glucose transport in this tissue is maintained mainly by the activity of the insulin-regulated glucose transporter GLUT4, although the ubiquitous GLUT1 glucose transporter is also present. Acute insulin treatment stimulates glucose transport in brown adipocytes largely by mediating translocation of GLUT4 from an intracellular compartment to the plasma membrane, a process involving the activation of PI3-kinase, Akt 1 and 2 isoforms and the atypical protein kinase $\mathrm{C}$ (PKC) $\zeta[14,15,16]$. Moreover, brown adipocytes are also a target for rosiglitazone action since they have high expression of PPAR $\gamma$, and rosiglitazone increased expression of the thermogenic uncoupling protein (UCP) 1 through binding to the PPAR response element described in the enhancer of the ucpl gene [17]. This cell model therefore provides an ideal system for investigating the mechanism by which TZDs increase insulin sensitivity.

\section{Materials and methods}

Materials. Rosiglitazone was kindly provided by Dr. S.A. Smith (Glaxo SmithKline, Harlow, UK). PD169316 and PD98059 were purchased from Calbiochem-Novabiochem International (La Jolla, Calif., USA). Myelin basic protein (MBP) was from Sigma Chemical (St. Louis, Mo., USA). Fetal calf serum, PBS, culture media and Trizol came from Invitrogen (Paisley, UK). GeneScreen nylon membranes were supplied by NEN Research Products (Boston, Mass., USA). Autoradiographic films were Kodak X-O-MAT/AR (Eastman Kodak, Rochester, N.Y., USA). The following were purchased from Amersham Biosciences (Little Chalfont, UK): 2-deoxy$\mathrm{D}\left[1-{ }^{3} \mathrm{H}\right]$-glucose $(444 \mathrm{GBq} / \mathrm{mmol}),\left(\alpha^{32} \mathrm{P}\right) \mathrm{dCTP},\left(\gamma^{32} \mathrm{P}\right) \mathrm{ATP}$, the multiprimer DNA-labelling system kit and protein G-sepharose. The cDNAs used as probes were GLUT4, GLUT1, UCP1 and fatty acid synthase (FAS) $[18,19,20]$. The rabbit antiGLUT1, anti-GLUT4 and anti-P-Ser antibodies were supplied by Chemicon (Tamacula, Calif., USA). The anti-phospho and anti- [Akt, p42/p44 mitogen-activated protein kinase (MAPK), p38MAPK] antibodies were from Cell Signaling (Beverly, Mass., USA). Antibodies against IRS-1, IRS-2 and P-Tyr (4G10) were from Upstate Biotechnology (Lake Placid, N.Y., USA). Antibodies against P-Tyr (PY20) "sc-508", IR $\beta$-chain "sc-09", IR $\alpha$-chain "sc-710", caveolin-1(N-20) "sc-894" and anti-PKC $\zeta$ "sc-216" were from Santa Cruz Laboratories (Palo Alto, Calif., USA). The anti-protein tyrosine phosphatase (PTP) $1 \mathrm{~B}$ and the anti-IR $\beta$-chain antibodies were from Oncogene Science (Uniondale, N.Y., USA). All other reagents used were of the purest grade available.

Cell culture. Fetal brown adipocytes were obtained from interscapular brown adipose tissue of 20-day Wistar rat fetuses and isolated by collagenase dispersion [21]. The isolated cells were plated in tissue culture dishes $\left(1 \times 10^{6}\right.$ cells per $60-\mathrm{mm}$ diameter dish, $4 \times 10^{6}$ cells per $100-\mathrm{mm}$ diameter dish) in minimal essential medium (MEM) with Earle's salts supplemented with $10 \%$ FCS, the presence of which was essential for the cells to attach to the plastic surface of the dishes. After 4 to $6 \mathrm{~h}$ of culture at $37^{\circ} \mathrm{C}$, the cells were rinsed twice with PBS and a $70 \%$ confluent monolayer was observed under inverse light microscopy. The cells were maintained for $20 \mathrm{~h}$ in a serum-free MEM medium supplemented with $0.2 \%$ (w/v) BSA and then further incubated for $24 \mathrm{~h}$ with or without $10 \mu \mathrm{mol} / \mathrm{l}$ rosiglitazone. In some experiments cells were treated with rosiglitazone with or without inhibitors [PD169316, $800 \mathrm{nmol} / \mathrm{l}$ (PD*) for p38MAPK and PD98059, $20 \mu \mathrm{mol} / \mathrm{l}$ (PD) for extracellular signal-regulated kinase]. At the end of the culture time some of the cells were stimulated for 5 to $30 \mathrm{~min}$ with different doses of insulin. The remaining cells were not stimulated. Wistar rats were obtained from the Complutense University Animal House. Principles of laboratory animal care were followed and the study was approved by the local ethics committee and carried out in accordance with the Declaration of Helsinki.

Measurement of glucose transport. Glucose transport was measured by incorporating 2-deoxy-D $\left[1-{ }^{3} \mathrm{H}\right]$-glucose into cells during the last $10 \mathrm{~min}$ of culture as described previously [14].

Subcellular fractionation. Cells were washed with ice-cold PBS and scraped in homogenisation buffer containing: $20 \mathrm{mmol} / \mathrm{l}$ Tris-HCl, $2 \mathrm{mmol} / \mathrm{l}$ EGTA, $2 \mathrm{mmol} / \mathrm{l}$ EDTA, $1 \mathrm{mmol} / \mathrm{l}$ phenylmethylsulfonyl fluoride, $10 \mathrm{mmol} / \mathrm{l} \beta$-mercaptoethanol, $10 \mu \mathrm{g} / \mathrm{ml}$ aprotinin, and $10 \mu \mathrm{g} / \mathrm{ml}$ leupeptin (pH 7.4). After a 10-min incubation period cells were homogenised with 30 strokes of a Dounce homogeniser using a tight-fitting pestle. Nuclei were pelleted by 5 min of centrifugation at $500 \mathrm{~g}$, and the low-speed supernatant was centrifuged for $30 \mathrm{~min}$ at $100,000 \mathrm{~g}$. The high-speed supernatant constituted the internal membrane fraction. The pellet was washed three times and extracted for $60 \mathrm{~min}$ in ice-cold homogenisation buffer containing $1 \%$ Triton $\mathrm{X}-100$. The Triton-soluble component (plasma membrane fraction) was separated from the Triton-insoluble material (cytoskeletal fraction) by centrifugation at $100,000 \mathrm{~g}$ for $15 \mathrm{~min}$. Internal and plasma membrane fractions were kept at $-70^{\circ} \mathrm{C}$ before protein quantification and western blotting with GLUT4 and Caveolin-1 antibodies [22].

RNA extraction and analysis. For northern-blot analysis of RNA at the end of the culture time the cells were washed twice with ice-cold PBS and lysed directly with Trizol according to 
a

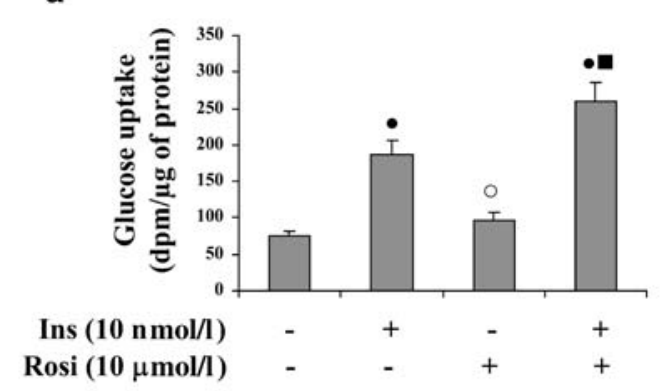

C

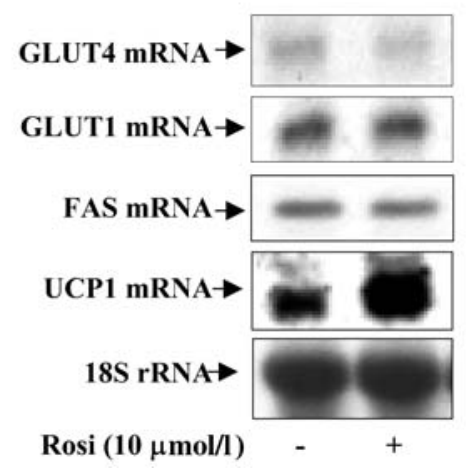

Fig. 1a-d. Rosiglitazone potentiates insulin-induced glucose uptake and GLUT4 translocation to plasma membrane in fetal brown adipocytes. Brown adipocytes (a) were pretreated or not pretreated with rosiglitazone for $24 \mathrm{~h}$ and stimulated or not stimulated with insulin for $30 \mathrm{~min}$. Glucose uptake was measured during the last $10 \mathrm{~min}$ of stimulation by incorporating 2deoxy-D $\left[1-{ }^{-3} \mathrm{H}\right]$-glucose. Results are expressed as disintegrations per minute per microgram of protein and are means \pm SEM $(n=10)$. Statistical significance was tested. Differences between values are shown by: $(0)$ with or without insulin; $(\bigcirc)$ rosiglitazone and control; and (ם) rosiglitazone plus insulin vs insulin; $(\bigcirc=0.0001 ; \quad \square=0.047 ; \bigcirc p=0.001$ ). Cells (b) were treated as in (a), and at the end of the culture time harvested and subjected to subcellular fractionation. Plasma membrane proteins were submitted to SDS/PAGE, blotted on to nylon membrane, immunodetected with anti-GLUT4 and anti-Caveolin-1 antibodies and developed with enhanced chemiluminiscence. Histograms from densitometric analysis are means $\pm \operatorname{SEM}(n=4)$, and statistical significance was tested as in (a). Brown adipocytes were treated (c) or not treated with rosiglitazone for $24 \mathrm{~h}$. Total RNA was submitted to northern-blot analysis and hybridised with labelled GLUT4, GLUT1, FAS and UCP1 cDNAs. Total protein was submitted (d) to western blotting and immunodetected with antibodies against GLUT4 and GLUT1 proteins

the manufacturer's protocol for total RNA isolation. Total cellular RNA $(15 \mu \mathrm{g})$ was submitted to northern blot analysis as described [23]. For serial hybridisation with different probes, the blots were stripped and then rehybridised as needed in each case. The membranes were subjected to autoradiography and the relative densities of the hybridisation signals were measured by densitometric scanning of the autoradiograms in a laser densitometer (Molecular Dynamics, Sunnyvale, Calif., USA).

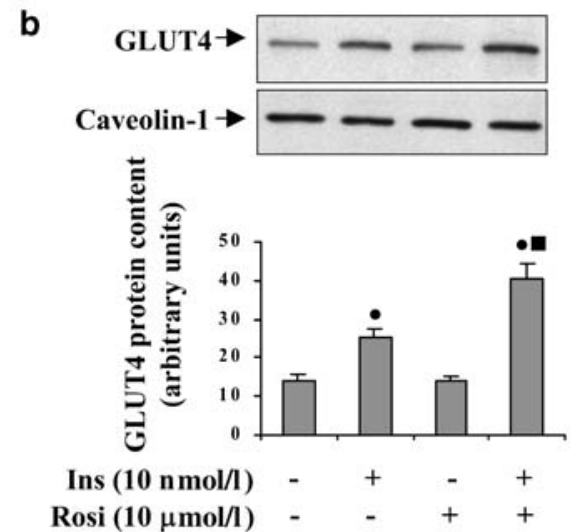

d

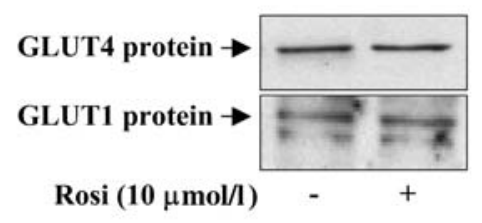

Immunoprecipitations. Cells were extracted with lysis buffer I containing: $10 \mathrm{mmol} / 1$ Tris, $50 \mathrm{mmol} / \mathrm{l} \mathrm{NaCl}, 1 \%$ Triton X-100, $5 \mathrm{mmol} / \mathrm{l}$ EDTA, $20 \mathrm{mmol} / \mathrm{l}$ sodium pyrophosphate, $50 \mathrm{mmol} / \mathrm{l}$ $\mathrm{NaF}, 100 \mu \mathrm{mol} / \mathrm{l} \mathrm{Na} \mathrm{VO}_{4}, 1 \mathrm{mmol} / \mathrm{l}$ phenylmethylsulfonyl fluoride ( $\mathrm{pH} 7.5$ ). They were then immunoprecipitated with different antibodies. PI3-kinase activity was measured in antiIRS-1, anti-IRS-2 or anti-P-(Tyr) (PY20 antibody) immunoprecipitates by in vitro phosphorylation of PI as described [22]. The association between IR and PTP1B was done by immunoprecipitation with anti-PTP1B antibody and immunoblotting with anti-P-Tyr (4G10) or anti-IR $\beta$-chain (Oncogene Science) or anti-PTP1B antibodies. To measure p38MAPK and PKC $\zeta$ activities, lysates were immunoprecipitated with antip38MAPK or anti-PKC $\zeta$ antibodies, and the immune complexes were used for in vitro phosphorylation of MBP, as described $[22,24]$.

PTP1B activity. Cells were cultured in a phosphate-free MEM medium, then lysed in lysis buffer II containing: $50 \mathrm{mmol} / \mathrm{l}$ Tris, $150 \mathrm{mmol} / \mathrm{l} \mathrm{NaCl}, 1 \%$ Triton X-100, 2 mmol/l EDTA (pH 7.5). Cell lysates were applied through a Sephadex G-25 column to remove the free phosphate present in the lysates. After protein determination, PTP1B activity was measured in terms of phosphate release using a synthetic Tyr phosphorylated peptide from IR $\beta$-chain regulatory autophosphorylation sites (Biomol Research Laboratories, Plymouth, UK) and the malachite green assay (Upstate Biotechnology).

Western blotting. Cells were lysed in lysis buffer I and cellular proteins $(30 \mu \mathrm{g})$ were submitted to SDS-PAGE, transferred to Immobilon membranes and blocked using 5\% non-fat dried milk in $10 \mathrm{mmol} / \mathrm{l}$ Tris- $\mathrm{HCl}$ and $150 \mathrm{mmol} / \mathrm{l} \mathrm{NaCl}(\mathrm{pH} 7.5)$. They were incubated overnight with several antibodies as indicated in each case in $0.05 \%$ Tween-20, $1 \%$ non-fat dried milk in $10 \mathrm{mmol} / \mathrm{l} \mathrm{Tris}-\mathrm{HCl}$ and $150 \mathrm{mmol} / \mathrm{l} \mathrm{NaCl}(\mathrm{pH}$ 7.5). Immunoreactive bands were visualised using the enhanced chemiluminiscence (ECL-Plus) western blotting protocol (Amersham). 
a

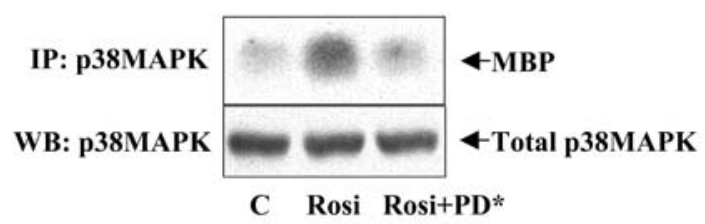

C

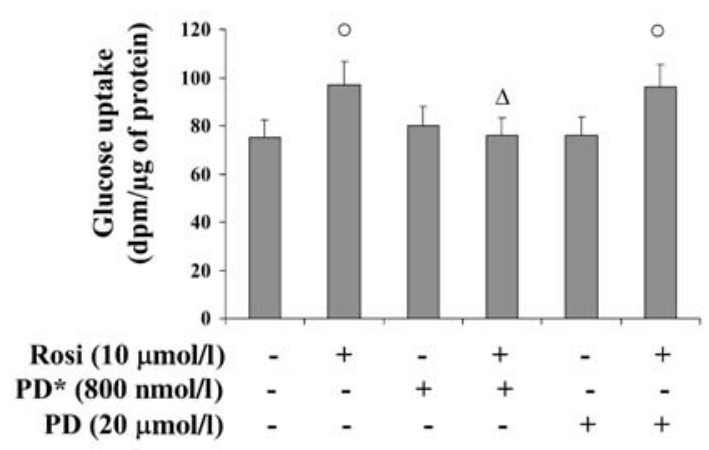

Fig. 2a-d. Rosiglitazone induction of basal glucose uptake depends on activation of $\mathrm{p} 38 \mathrm{MAPK}$ but not on $\mathrm{p} 44 / \mathrm{p} 42 \mathrm{MAPK}$. Brown adipocytes were cultured for $6 \mathrm{~h}$ with or without rosiglitazone and without or with PD98059 (PD) or PD169316 $\left(\mathrm{PD}^{*}\right)(\mathbf{a}, \mathbf{b})$. Cell lysates were immunoprecipitated with antip38MAPK antibody and assayed for kinase activity using MBP as substrate (a) or submitted to western blotting and immunodetected with anti-phospho- or anti-(p44/p42MAPK) antibodies (b). Cells were cultured for $24 \mathrm{~h}$ with or without rosiglitazone and with or without PD or PD* (c). After $24 \mathrm{~h}$, glucose uptake was measured for $10 \mathrm{~min}$ by incorporating 2-deoxy-D $\left[1-{ }^{3} \mathrm{H}\right]$-glucose. Results are expressed as disintegrations per min per microgram of protein and are means \pm SEM $(n=10)$. Statistical significance was tested. Differences between values are shown by: $(\bigcirc)$ presence of rosiglitazone vs control; $(\triangle)$ presence of rosiglitazone plus PD* vs rosiglitazone. Cells (d) were cultured as in (c), and after $24 \mathrm{~h}$ stimulated with insulin for $30 \mathrm{~min}$. Glucose uptake was measured in the last $10 \mathrm{~min}$ of stimulation. Results are expressed as in (c). Statistical significance was tested. Differences between values for the presence and absence of rosiglitazone are shown by (ם); $(\bigcirc, \triangle, \square) p<0.05$

Data analysis. Results are means \pm SEM ( $n=4$ to 10 ) for duplicate dishes from four to ten independent experiments. Statistical significance was tested with ANOVA followed by the protected least significant different test. A $p$ value of less than 0.05 was considered significant. In experiments using X-ray films (Hyperfilm), different exposure times were used to ensure that bands were not saturated.

\section{Results}

Fetal rat brown adipocytes isolated and cultured for $44 \mathrm{~h}$ in a serum-free medium $(0.2 \%$ BSA-MEM $)$ b

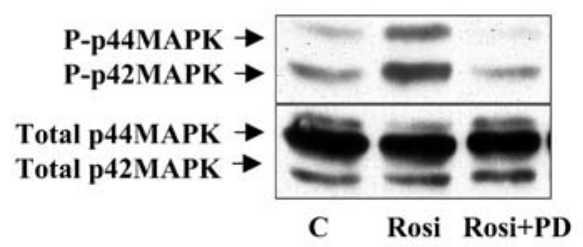

d

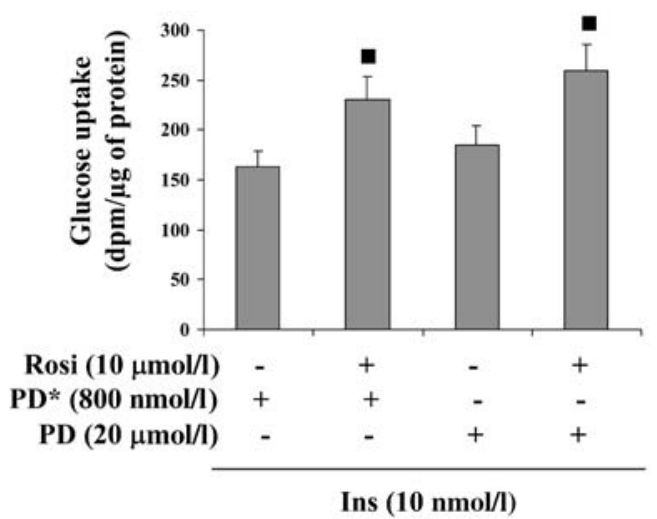

maintained the expression of characteristic lipogenic and thermogenic genes such as FAS and UCP1, as well as expressed the insulin-regulated glucose transporter GLUT4 together with the ubiquitous glucose transporter GLUT1 (Fig. 1c). Under these conditions, treatment for $30 \mathrm{~min}$ with $10 \mathrm{nmol} / \mathrm{l}$ insulin increased glucose uptake 2.5-fold compared with untreated cells (Fig. 1a). When cells were cultured for $20 \mathrm{~h}$ in a serum-free medium and pretreated for $24 \mathrm{~h}$ with $10 \mu \mathrm{mol} / \mathrm{l}$ rosiglitazone, insulin stimulation of glucose uptake was much higher (3.5-fold higher than basal and $40 \%$ higher than the insulin-stimulated value). Rosiglitazone per se also produced a $25 \%$ increase over basal glucose uptake (Figs. 1a, 2c).

As insulin stimulation of glucose transport is mediated by the translocation of GLUT4 to the plasma membrane, we established the permissive effect of rosiglitazone on glucose uptake by examining GLUT4 translocation. Insulin increased GLUT4 translocation to plasma membrane fraction by twofold, but produced a threefold translocation when brown adipocytes were pretreated with rosiglitazone for $24 \mathrm{~h}$. Caveolin-1, which was used as a marker protein of the plasma membrane, remained essentially unchanged under the different treatments used (Fig. 1b). To validate if the increase in insulin-induced glucose uptake and GLUT4 translocation produced by rosiglitazone was dependent on GLUT gene expression, the levels of these mRNAs were analysed together with lipogenic and thermogenic markers (Fig. 1c). Rosiglitazone treatment for $24 \mathrm{~h}$ did not change GLUT4 or GLUT1 expression at the mRNA or protein level (Fig. 1c, d). FAS mRNA levels also remained unchanged, although expression of UCP1, a target gene for rosiglitazone, 
a
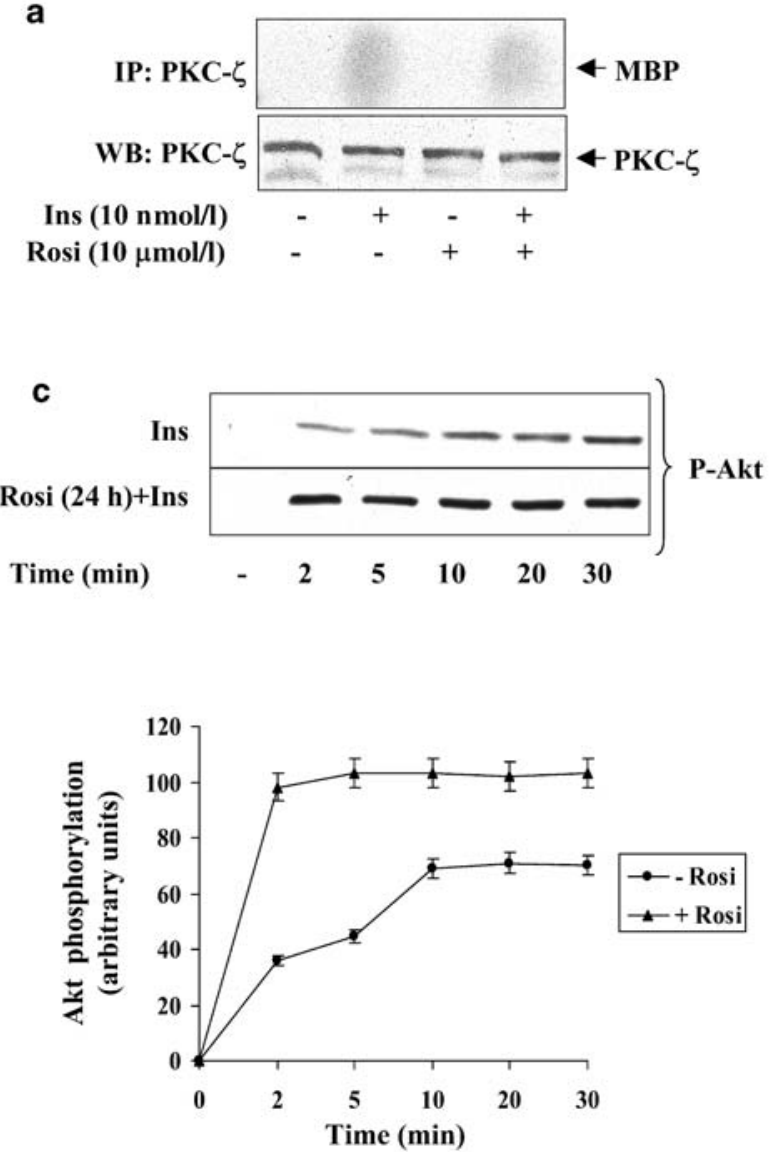

Fig. 3a-d. Rosiglitazone potentiates insulin activation of Akt but not of $\mathrm{PKC} \zeta$ in brown adipocytes. Brown adipocytes $(\mathbf{a}, \mathbf{b})$ were pretreated or not pretreated with rosiglitazone for $24 \mathrm{~h}$. After $24 \mathrm{~h}$ cells were stimulated with insulin for $5 \mathrm{~min}$. Cell lysates were immunoprecipitated with anti-PKC $\zeta$ antibody and assayed for kinase activity using MBP as substrate (a). Total protein $(30 \mu \mathrm{g})$ was submitted to western blotting and immunodetected with anti-phospho- or anti-(Akt) antibodies (b). Brown adipocytes (c, d) were pretreated or not, as in (a) and (b), stimulated for 2 to $30 \mathrm{~min}$ with $10 \mathrm{nmol} / \mathrm{l}$ insulin (c) or for 5 min with 1 to $100 \mathrm{nmol} / \mathrm{l}$ insulin (d), and submitted to western blotting with an anti-phospho-Akt (Ser 473) antibody. Diagrams from densitometric analysis are means \pm SEM $(n=4)$

was dramatically induced in these experimental conditions (Fig. 1c).

The $25 \%$ increase in basal glucose uptake induced by rosiglitazone without a detectable increase in GLUT4 translocation could result from activation of the glucose transporter, an event that has been related to p38MAPK activation. To explore the possible involvement of p38MAPK or extracellular signal-regulated kinase on the induction of glucose uptake by rosiglitazone, either with or without insulin, we used chemical inhibitors for those kinases (Fig. 2a, b).

The rosiglitazone-induced increase in basal glucose uptake was inhibited by PD* but not by PD (Fig. 2c). A $40 \%$ higher increase in insulin-stimulated glucose uptake was produced by pretreatment with rosiglita-

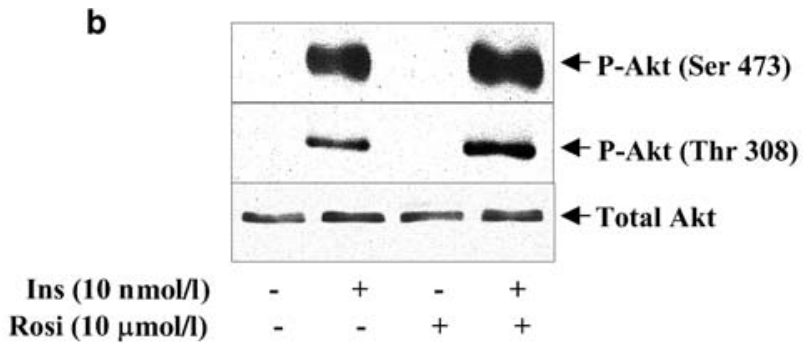

d
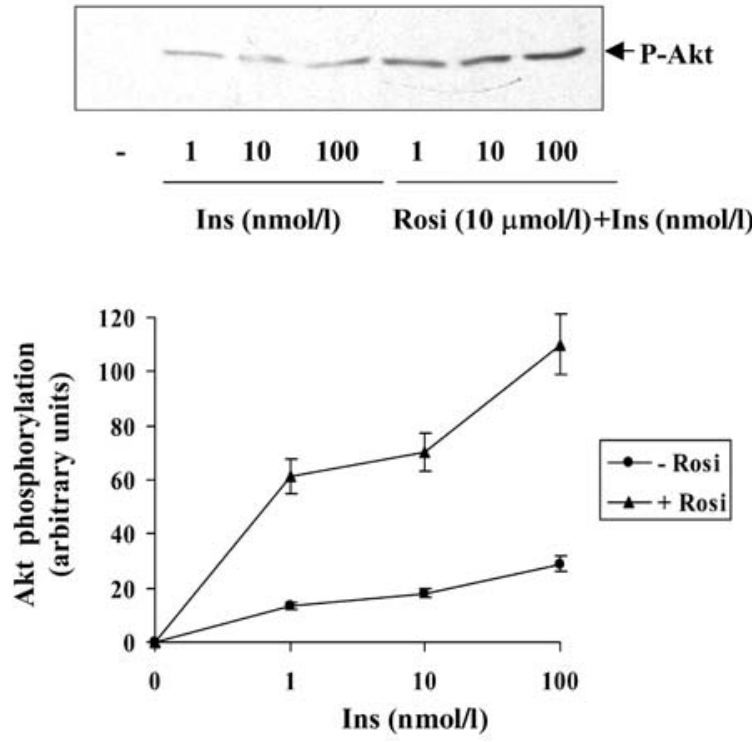

zone, regardless of whether inhibitors were present or not (Fig. 2d).

We also examined whether rosiglitazone activates the main insulin signalling pathways (Akt and $\mathrm{PKC} \zeta$ ) proposed to mediate translocation of GLUT4 from an intracellular compartment to the plasma membrane in brown adipocytes (Fig. 3). Brown adipocytes expressed $\mathrm{PKC} \zeta$ and its enzymatic activity was analysed in the anti-PKC $\zeta$ immune complexes. As shown in Fig. 3a, insulin-induced MBP phosphorylation was not modified by pretreatment with rosiglitazone. Western blotting with the anti-phospho Akt antibodies for regulatory residues Ser 473 and Thr 308, and with the total Akt antibody showed that upon insulin stimulation Akt was highly Ser phosphorylated and to a lower extent Thr phosphorylated. Both effects were increased by pretreatment with rosiglitazone, although rosiglitazone per se did not produce Akt activation (Fig. 3b). These data indicate that the increase in insulin-induced glucose uptake produced by rosiglitazone was parallel to the increase in insulin-activation of Akt.

We further explored this sensitising effect of rosiglitazone on insulin stimulation of Akt in kinetics and dose-response studies. Insulin-optimal phosphorylation of Akt occurred after treatment for $10 \mathrm{~min}$, although 2 min of insulin stimulation was enough for maximal Akt activation in cells pretreated with rosiglitazone (Fig. 3c). Moreover, the potentiation of Akt 
a
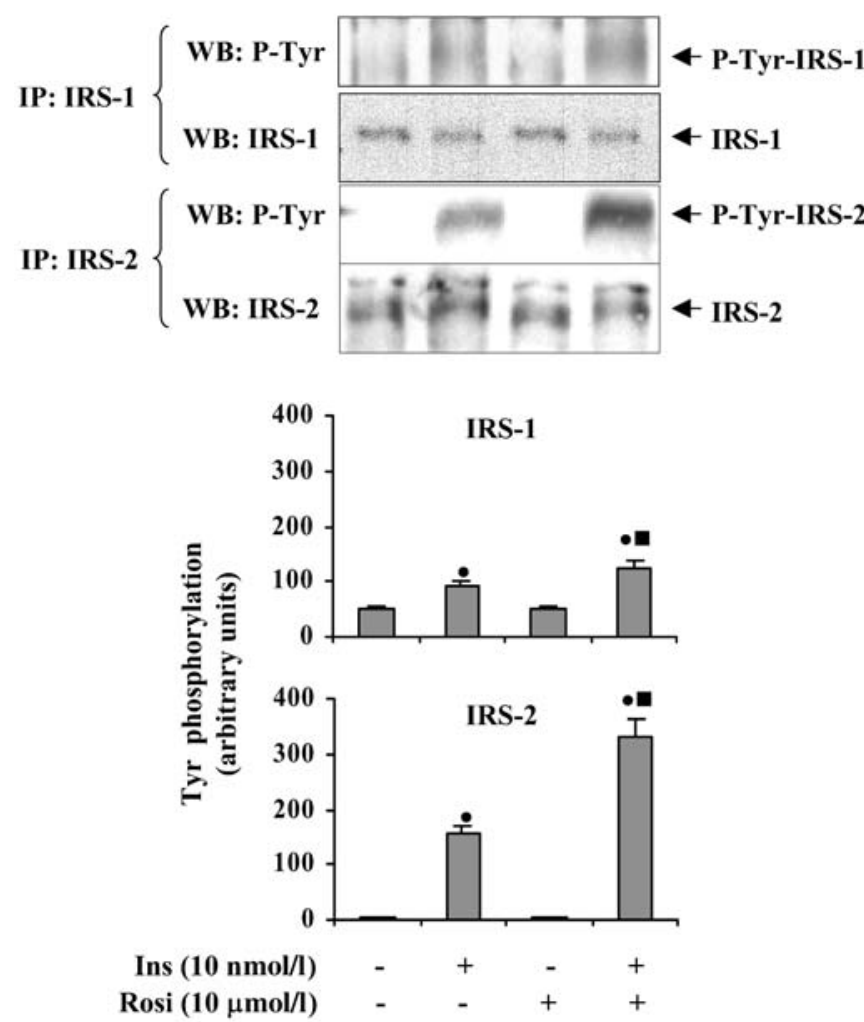

Fig. 4a, b. Rosiglitazone increases insulin-induced Tyr phosphorylation of IRS-1 and IRS-2 and its associated PI3-kinase activity in brown adipocytes. Cells were cultured as in Fig. 3a and lysates immunoprecipitated with anti-IRS-1 or anti-IRS-2 antibodies and assayed for Tyr phosphorylation (a) or PI3-kinase activity (b). Histograms from densitometric analysis are means \pm SEM $(n=4)$. Statistical significance was tested as in Fig. 1a

phosphorylation seen in the presence of rosiglitazone was maintained after $30 \mathrm{~min}$ of insulin stimulation. This is consistent with the data on glucose uptake and GLUT4 translocation at this time point. The sensitising effect of rosiglitazone on Akt phosphorylation by insulin was detected at all the insulin doses tested (Fig. 3d).

We next examined whether insulin sensitisation by rosiglitazone at the Akt level is a result of upstream Akt potentiation. Tyr phosphorylation and PI3-kinase activity associated with IRSs were measured in the immune complexes after immunoprecipitation with either the anti-IRS-1 or anti-IRS-2 antibodies (Fig. 4). Rosiglitazone potentiated insulin-stimulated Tyr phosphorylation of IRS-1 and to a greater extent of IRS-2, without modifying expression of IRSs (Fig. 4a). Moreover, insulin-induced PI3-kinase activity in P-Tyr or IRS-1 or IRS-2 immunoprecipitates noticeably increased under pretreatment with rosiglitazone, this effect being again more prominent at the IRS-2 level (Fig. 4b). b
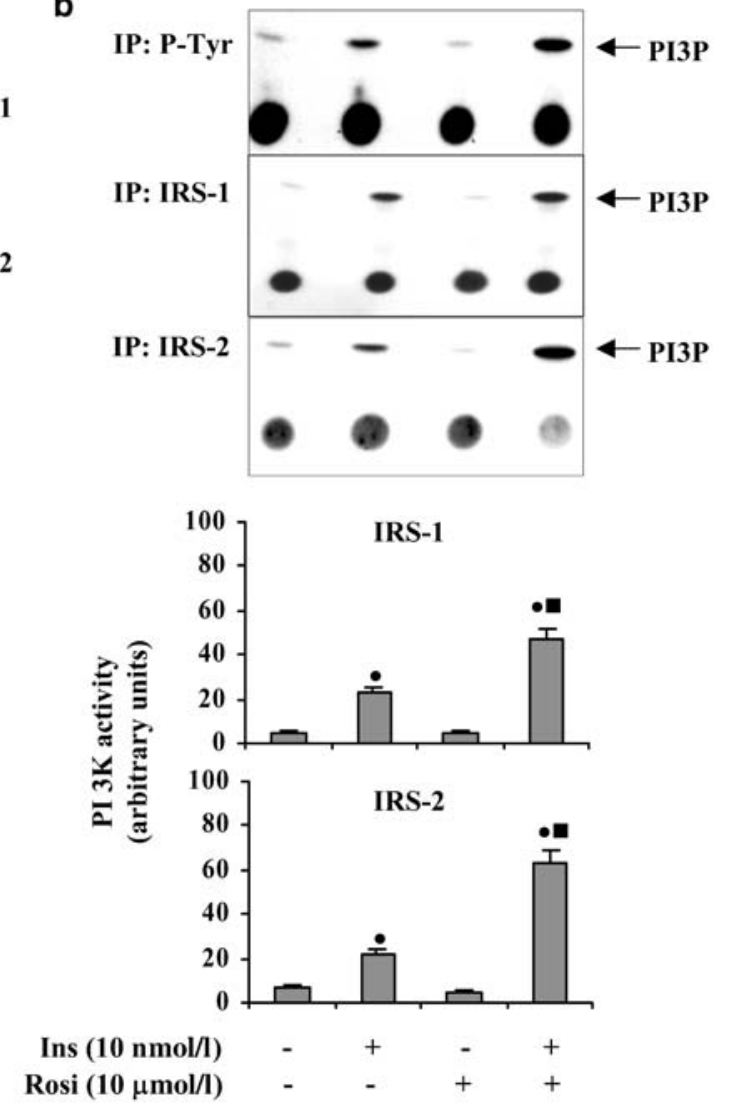

This prompted us to explore the effects of rosiglitazone on insulin signalling at the level of IR. Direct western blotting with anti-IR $\beta$-chain or $\alpha$-chain antibodies showed that pretreatment with rosiglitazone doubled the amount of IR compared with cells cultured without rosiglitazone (Fig. 5a). This also happened in IR immunoprecipitates, where insulin-induced Tyr phosphorylation of IR after 5 or $30 \mathrm{~min}$ increased after pretreatment with rosiglitazone (Fig. 5b). Stimulation with insulin for 5 min not only increased Tyr phosphorylation of IR but also produced Ser phosphorylation of the IR, as shown in immunoprecipitates with P-Ser antibodies and western blotting with IR $\beta$ chain antibody. Surprisingly, Ser phosphorylation of IR was lower in brown adipocytes pretreated with rosiglitazone and stimulated with insulin, resulting in a reciprocal increase in IR Tyr phosphorylation (Fig. 5c). This suggests that rosiglitazone modulates insulin signalling by acting through phosphatases at the level of the IR. Of these PTP1B has been shown to be expressed in insulin-sensitive tissues and also to bind to and dephosphorylate IR and IRS-1. The IR coprecipitated with PTP1B in lysates from untreated cells, this interaction being increased after insulin stimulation. Immunoblotting with anti-P-Tyr antibody showed that PTP1B was phosphorylated under insulin stimulation, but Tyr phosphorylation of IR remained low. However, under insulin stimulation after pretreatment with rosiglitazone an increase was observed in IR association with PTP1B and especially in Tyr 
a

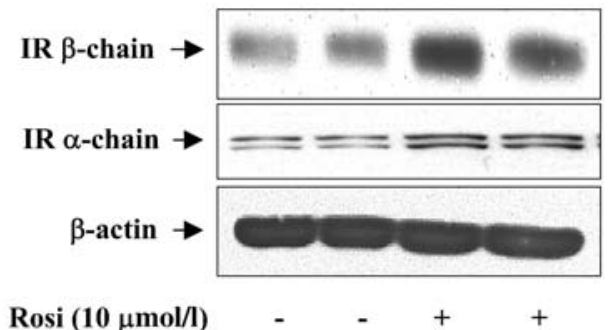

b
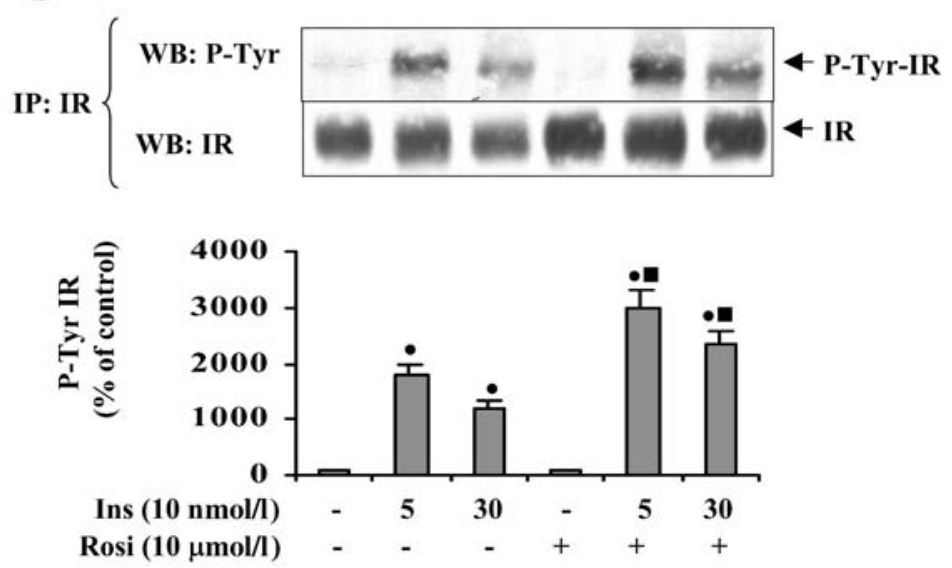

Fig. 5a-c. Rosiglitazone increases IR expression and insulinstimulated IR $\beta$-chain Tyr phosphorylation. Cells were cultured as in Fig. 1c and lysates submitted to direct western blotting with antibodies against the $\beta$-chain and $\alpha$-chain of IR or against $\beta$-actin (a). Histograms from densitometric analysis are means $\pm \operatorname{SEM}(n=4)$. Statistical significance was tested. Differences between values for the presence of rosiglitazone vs control are represented by $(\bullet)$. Brown adipocytes were pretreated or not pretreated with rosiglitazone for $24 \mathrm{~h}$ and then stimulated with insulin for 5 or $30 \mathrm{~min}$ (b). Cell lysates were immunoprecipitated with antibodies against IR and immunoblotted with anti-P-Tyr (4G10) antibody or with the antibody against the $\beta$-chain of IR. Histograms from densitometric analysis are means \pm SEM $(n=4)$. Differences between values are shown by: (@) presence of insulin vs control; and (ם) presence of rosiglitazone plus insulin vs insulin. Cell lysates were immunoprecipitated with antibodies against P-Ser and immunoblotted with the antibody against the $\beta$-chain of the IR (c). Histograms from densitometric analysis are means \pm SEM $(n=4)$. Differences between values are shown by: $(-)$ presence of insulin vs control; and $(\triangle)$ presence of rosiglitazone plus insulin vs insulin; $(\boldsymbol{\bullet}, \triangle) p<0.05$

phosphorylation of PTP1B and IR. Equal amounts of PTP1B were present in the immunoprecipitates, as seen upon stripping and reblotting with an anti-PTP1B antibody (Fig. 6a). The amount of PTP1B or other phosphatases such as SHP2 or PTEN remained unaltered by rosiglitazone treatment as assessed by direct western blotting (Fig. 6b).

Given the above, our last experiment was an assay of PTP1B activity using a Tyr phosphorylated peptide

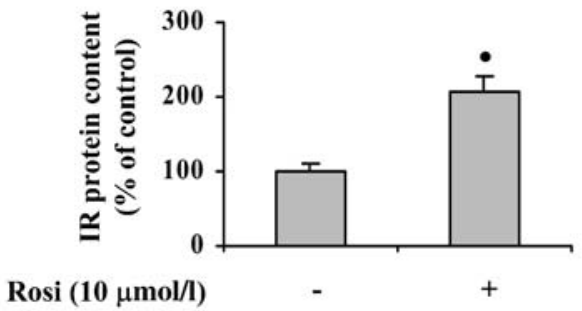

$\operatorname{Rosi}(10 \mu \mathrm{mol} / \mathrm{l})$
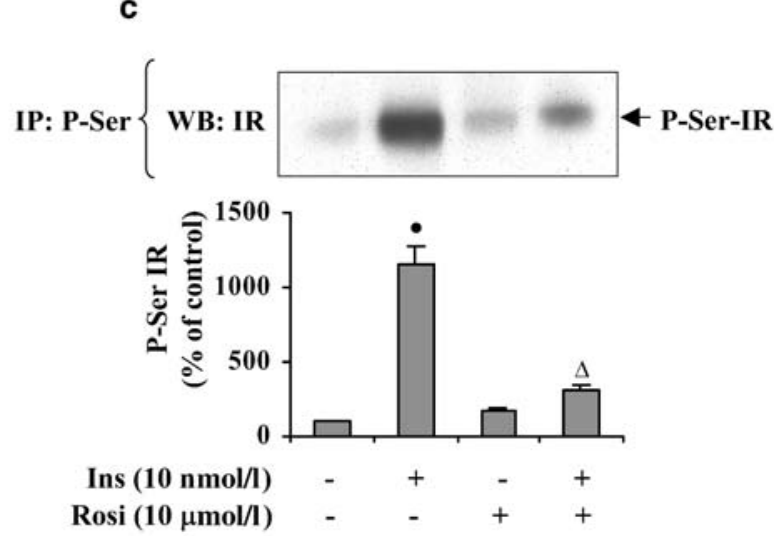

including the regulatory autophosphorylation sites (PTyr1146, 1150 and 1151) of the IR $\beta$-chain subunit as substrate (Fig. 6c). Insulin stimulation for $5 \mathrm{~min}$ increased PTP1B activity, which was impaired in cells pretreated with rosiglitazone. This effect represents a second mechanism, by which rosiglitazone could contribute to increasing Tyr phosphorylation of IR, as detected in the PTP1B and IR immunoprecipitates (Fig. 5, Fig. 6).

\section{Discussion}

In recent years our laboratory has established that fetal brown adipocytes constitute a suitable cell model to study insulin signalling and glucose uptake under physiological and insulin-resistant conditions. These cells have numerous high-affinity insulin binding sites, and glucose transport is maintained mainly by the insulin-regulated glucose transporter GLUT4 [21, 25]. Brown adipocytes are also a target for the action of TZDs, as they show high expression of PPAR $\gamma$ [17]. We have recently proposed that rosiglitazone increases UCP1 gene expression by acting as a PPAR/RXR agonist and also by activating p38MAPK [26]. In this work we found that rosiglitazone increased basal and insulin-stimulated glucose uptake by different mechanisms. Chronic treatment with rosiglitazone produced a modest but significant increase in basal glucose uptake. This effect was not the result of changes in the expression of the GLUT4 gene, but was dependent on 


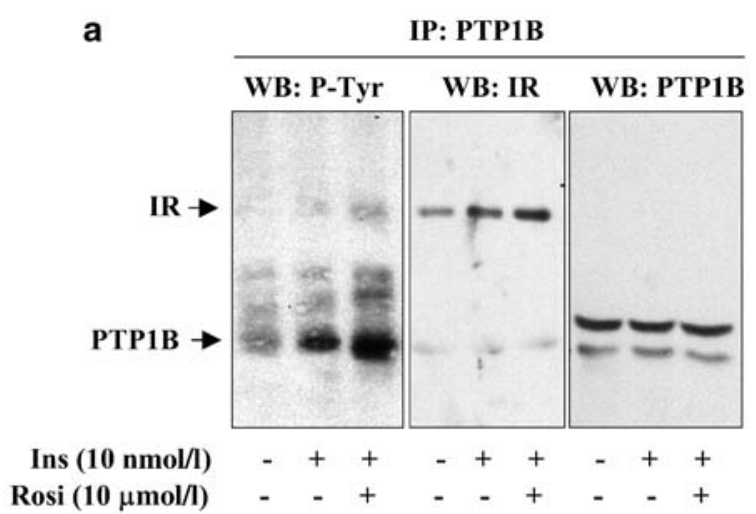

b

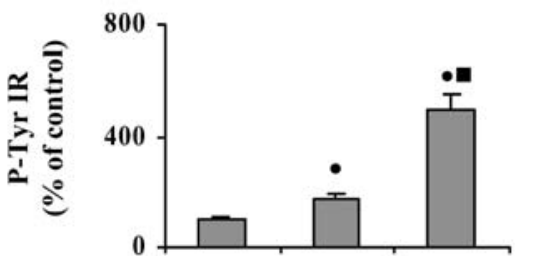

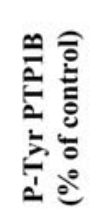
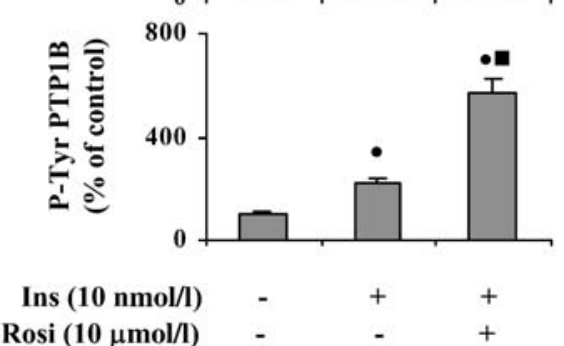

Fig. 6a-c. Rosiglitazone increases insulin-induced Tyr phosphorylation of IR and PTP1B in PTP1B immunoprecipitates and impairs insulin activation of PTP1B activity. Cells were cultured as in Fig. 3a and lysates were immunoprecipitated with antibodies against PTP1B and immunoblotted with antiP-Tyr antibody or with the antibody against the $\beta$-chain of the IR or with anti-PTP1B antibody (a). Histograms from densitometric analysis are means $\pm \operatorname{SEM}(n=4)$. Statistical significance was tested as in Fig. 1a. Direct western blotting against several phosphatases in cell lysates from cells cultured with or without rosiglitazone are shown (b). Cells cultured in a phosphate-free medium were treated as described in (a), and at the end of the culture time PTP1B activity was measured (c). Results are expressed as a percentage of stimulation of phosphatase activity compared with control cells and are means \pm SEM $(n=4)$. Statistical significance was tested as described. Differences between values are shown by: $(\mathbf{)}$ presence of insulin vs control; and $\triangle$, presence of rosiglitazone plus insulin vs insulin; $(\bullet, \triangle) p<0.05$

p38MAPK activation. The results of the present study show that the effects of rosiglitazone on glucose uptake are extragenic and mediated by activation of p38MAPK. Since rosiglitazone did not modify GLUT4 translocation to the plasma membrane, its effect on glucose uptake could be attributed to activation of the glucose transporter. This would be consistent with the previously described activation of GLUT4 by p38MAPK in response to insulin [27, 28]. The same team has also described the activation of GLUT4 by p38MAPK in response to lipoic acid [29].

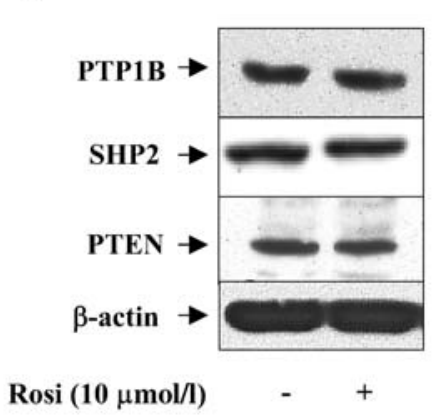

c

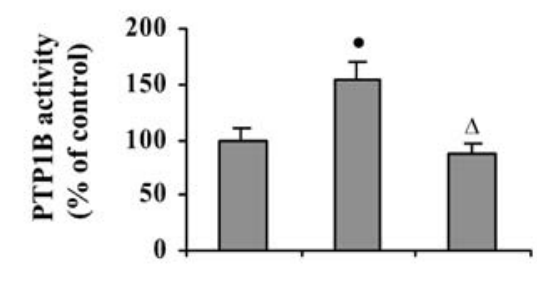

$\begin{array}{rlll}\text { Ins }(10 \mathrm{nmol} / \mathrm{l}) & - & + & + \\ \operatorname{Rosi}(10 \mu \mathrm{mol} / \mathrm{l}) & - & - & +\end{array}$
The increase in insulin-stimulated glucose uptake produced by treatment with rosiglitazone $(40 \%)$ was still observable in the presence of the p38MAPK inhibitor PD169316, indicating that this effect was independent of GLUT4 activation. Although rosiglitazone treatment did not change expression of GLUT4 or GLUT1 at the mRNA or protein level, it did increase insulin-induced GLUT4 translocation from an intracellular compartment to the plasma membrane. Glucose uptake has been found to be increased by TZDs in isolated adipocytes and in 3T3-L1 adipocytes due to increases in GLUT4 or GLUT1 expression [4, 10]. We have shown that this effect in primary brown adipocytes is a result of GLUT4 translocation to the plasma membrane. Two kinases, Akt and PKC $\zeta$, can contribute to GLUT4 translocation by insulin in these cells $[14,15,16]$. Rosiglitazone potentiated insulin activation of Akt at all the insulin doses tested and modified the kinetic of induction, whereas PKC $\zeta$ was not affected. Our data are in contrast to previous findings $[30,31]$, where PKC $\zeta$ but not Akt was activated in adipocytes under rosiglitazone stimulation. In agreement with our results, insulin sensitising effects of TZDs at the level of Akt have been reported in several models of obesity and diabetes in vivo and in vitro supporting the importance of this kinase, especially the isoform Akt 2, in maintaining glucose disposal or glucose uptake $[3,5]$. This effect could be a consequence of insulin sensitisation by rosiglitazone treatment at this level or at an upper level. In this regard 
we detected increased insulin-stimulated Tyr phosphorylation and PI3-kinase activity associated with IRS-1 and IRS-2. This sensitising effect was more potent at the level of IRS-2, in agreement with our previous findings on the more prevalent implication of this docking protein in glucose uptake, both in brown adipocytes and in other insulin-responsive cells [22, 32, 33].

Our results also indicate that treatment with rosiglitazone resulted in a marked increase in the sensitivity of the IR for insulin. This is a consequence of two convergent mechanisms: (i) increased IR expression (presumably dependent on activation of nuclear receptors); and (ii) activation of IR (probably extragenic effects). Regarding the first, rosiglitazone could bind to specific nuclear receptors to activate the transcription of IR, in a similar fashion to that described for UCP1 [26]. This effect does not seem to be related to the differentiation-inducing properties of rosiglitazone, because expression of the adipogenic gene FAS remains unmodified. Although no PPAR response element in the IR promoter has yet been identified, a decrease in IR expression has been described in adipocytes overexpressing a dominant-negative mutant of PPAR $\gamma$ [34].

Despite other evidence of the effects of TZDs on expression of IRS-2, p85-PI3-kinase and Cbl-associated protein $[7,8,9]$, we only detected changes in the expression of IR. The higher amount of IR detected under rosiglitazone treatment could explain the increased Tyr phosphorylation observed in IR immunoprecipitates. However, under these conditions a decrease in Ser phosphorylation of IR was detected, a situation that also favours Tyr phosphorylation of IR, indicating the possibility of an additional extragenic mechanism. It has been proposed that the kinase domain of IR acts as a dual specificity kinase, making phosphorylation of its own Ser and Tyr residues possible [35], as we detected in brown adipocytes. Moreover, rosiglitazone could modulate insulin signalling by acting through phosphatases at the level of the IR. In this regard we found that insulin induced IR association with PTP1B, Tyr phosphorylation of PTP1B and increased PTP1B activity, resulting in a low Tyr phosphorylated IR $\beta$-chain in PTP1B immunoprecipitates. However, rosiglitazone increased insulin-induced Tyr phosphorylation of PTP1B and IR $\beta$-chain and impaired PTP1B activation by insulin. Phosphorylation of PTP1B at three Tyr residues seems to be necessary for association with the IR and activation of the PTP1B [36, 37], although extensive phosphorylation such as that produced by rosiglitazone could phosphorylate motifs in the C-terminal domain, in close proximity to the active site, thereby inactivating the phosphatase [38]. This reduction of PTP1B activity by rosiglitazone can contribute to increases in the signalling through IR in fetal primary brown adipocytes in a similar fashion to that detected in IGFIR-deficient brown adipocytes [39], or in $\mathrm{PTP}_{\mathrm{B}} \mathrm{B}^{-/}$fibroblasts exhibiting increased Tyr phosphorylation of EGFR and PDGFR after growth factor treatment [40]. However, we cannot rule out the implication of other phosphatases in insulin sensitisation by rosiglitazone, either at the IR level or downstream in the insulin signalling cascade.

Although the precise mechanism by which TZDs enhance insulin sensitivity in vivo is not known, it is believed to be mediated by their effects as PPAR $\gamma$ agonists on fat tissues. However, the potentiation of glucose uptake by rosiglitazone in 3T3-L1 adipocytes expressing a PPAR $\gamma$ dominant-negative mutant has been described [10], suggesting the potential implication of PPAR $\gamma$-independent mechanisms which could also operate in skeletal muscle, as we propose for brown adipocytes through modulation of protein kinases and phosphatases. In this context the potentiation of insulin signalling by TZDs has also been described in the soleus muscle, as well as in $\mathrm{C} 2 \mathrm{C} 12$ myotubes, i.e. cells which hardly express PPAR $\gamma[41,42]$.

In conclusion, rosiglitazone improved insulin sensitivity at the level of the IR. This increased IR expression and activation, and subsequently the insulin signalling cascade IRS-2/PI3-kinase/Akt, leading to potentiation of insulin-induced GLUT4 translocation and glucose uptake.

Acknowledgements. T. Teruel is the recipient of a postdoctoral fellowship from the Comunidad Autonoma de Madrid (City of Madrid). R. Hernandez is the recipient of a postgraduate fellowship from the Ministry of Education and Culture, Spain. This work was supported by BMC2002-01322 from the Ministry of Science and Technology, Spain. We thank COST/B17 Action on "Insulin resistance, obesity and diabetes mellitus in the elderly" from the European Commission.

\section{References}

1. Olefsky JM (2000) Treatment of insulin resistance with peroxisome proliferator-activated receptor gamma agonists. J Clin Invest 106:467-472

2. Olefsky JM, Saltiel AR (2000) PPAR gamma and the treatment of insulin resistance. Trends Endocrinol Metab 11: 362-368

3. Meyer MM, Levin K, Grimmsmann T et al. (2002) Troglitazone treatment increases protein kinase B phosphorylation in skeletal muscle of normoglycemic subjects at risk for the development of type 2 diabetes. Diabetes 51:26912697

4. Ciaraldi TP, Kong AP, Chu NV et al. (2002) Regulation of glucose transport and insulin signaling by troglitazone or metformin in adipose tissue of type 2 diabetic subjects. Diabetes 51:30-36

5. Jiang G, Dallas-Yang Q, Li Z et al. (2002) Potentiation of insulin signaling in tissues of Zucker obese rats after acute and long-term treatment with PPARgamma agonists. Diabetes 51:2412-2419

6. Cho H, Mu J, Kim JK et al. (2001) Insulin resistance and a diabetes mellitus-like syndrome in mice lacking the protein kinase Akt2 (PKB beta). Science 292:1728-1731 
7. Smith U, Gogg S, Johansson A, Olausson T, Rotter V, Svalstedt B (2001) Thiazolidinediones (PPARgamma agonists) but not PPARalpha agonists increase IRS-2 gene expression in 3T3-L1 and human adipocytes. FASEB J $15: 215-220$

8. Rieusset J, Auwerx J, Vidal H (1999) Regulation of gene expression by activation of the peroxisome proliferator-activated receptor gamma with rosiglitazone (BRL 49653) in human adipocytes. Biochem Biophys Res Commun 265: 265-271

9. Camp HS, Li O, Wise SC et al. (2000) Differential activation of peroxisome proliferator-activated receptor-gamma by troglitazone and rosiglitazone. Diabetes 49:539-547

10. Nugent C, Prins JB, Whitehead JP et al. (2001) Potentiation of glucose uptake in 3T3-L1 adipocytes by PPAR gamma agonists is maintained in cells expressing a PPAR gamma dominant-negative mutant: evidence for selectivity in the downstream responses to PPAR gamma activation. Mol Endocrinol 15:1729-1738

11. Teruel T, Valverde AM, Benito M, Lorenzo M (1996) Insulin-like growth factor I and insulin induce adipogenic-related gene expression in fetal brown adipocyte primary cultures. Biochem J 319:627-632

12. Teruel T, Valverde AM, Navarro P, Benito M, Lorenzo M (1998) Inhibition of PI 3-kinase and RAS blocks IGF-I and insulin-induced uncoupling protein 1 gene expression in brown adipocytes. J Cell Physiol 176:99-109

13. Navarro P, Valverde AM, Benito M, Lorenzo M (1998) Insulin/IGF-I rescues immortalized brown adipocytes from apoptosis down-regulating $\mathrm{Bcl}-\mathrm{xS}$ expression, in a PI 3-kinase- and map kinase-dependent manner. Exp Cell Res 243:213-221

14. Hernandez R, Teruel T, Lorenzo M (2001) Akt mediates insulin induction of glucose uptake and up-regulation of GLUT4 gene expression in brown adipocytes. FEBS Lett 494:225-231

15. Valverde AM, Lorenzo M, Navarro P, Mur C, Benito M (2000) Okadaic acid inhibits insulin-induced glucose transport in fetal brown adipocytes in an Akt-independent and protein kinase $\mathrm{C}$ zeta-dependent manner. FEBS Lett 472:153-158

16. Lorenzo M, Teruel T, Hernandez R, Kayali AG, Webster NJ (2002) PLCgamma participates in insulin stimulation of glucose uptake through activation of PKCzeta in brown adipocytes. Exp Cell Res 278:146-157

17. Teruel T, Clapham JC, Smith SA (1999) PPARalpha activation by Wy 14643 induces transactivation of the rat UCP-1 promoter without increasing UCP-1 mRNA levels and attenuates PPARgamma-mediated increases in UCP-1 mRNA levels induced by rosiglitazone in fetal rat brown adipocytes. Biochem Biophys Res Commun 264:311315

18. Birnbaum MJ (1989) Identification of a novel gene encoding an insulin-responsive glucose transporter protein. Cell 57:305-315

19. Bouillaud F, Ricquier D, Thibault J, Weissenbach J (1985) Molecular approach to thermogenesis in brown adipose tissue: cDNA cloning of the mitochondrial uncoupling protein. Proc Natl Acad Sci U S A 82:445-448

20. Amy CM, Witkowski A, Naggert J, Williams B, Randhawa Z, Smith S (1989) Molecular cloning and sequencing of cDNAs encoding the entire rat fatty acid synthase. Proc Natl Acad Sci USA 86:3114-3118

21. Lorenzo M, Valverde AM, Teruel T, Benito M (1993) IGF-I is a mitogen involved in differentiation-related gene expression in fetal rat brown adipocytes. J Cell Biol 123: $1567-1575$
22. Teruel T, Hernandez R, Lorenzo M (2001) Ceramide mediates insulin resistance by tumor necrosis factor-alpha in brown adipocytes by maintaining Akt in an inactive dephosphorylated state. Diabetes 50:2563-2571

23. Teruel T, Valverde AM, Alvarez A, Benito M, Lorenzo M (1995) Differentiation of rat brown adipocytes during late foetal development: role of insulin-like growth factor I. Biochem J 310:771-776

24. Conejo R, De Alvaro C, Benito M, Cuadrado A, Lorenzo M (2002) Insulin restores differentiation of Ras-transformed $\mathrm{C} 2 \mathrm{C} 12$ myoblasts by inducing NF-kappaB through an AKT/P70S6K/p38-MAPK pathway. Oncogene 21:3739_ 3753

25. Valverde AM, Navarro P, Teruel T, Conejo R, Benito M, Lorenzo M (1999) Insulin and insulin-like growth factor I up-regulate GLUT4 gene expression in fetal brown adipocytes, in a phosphoinositide 3-kinase-dependent manner. Biochem J 337:397-405

26. Teruel T, Hernandez R, Benito M, Lorenzo M (2003) Rosiglitazone and retinoic acid induce uncoupling protein1 (UCP-1) in a p38 mitogen-activated protein kinase-dependent manner in fetal primary brown adipocytes. J Biol Chem 278:263-269

27. Somwar R, Kim DY, Sweeney G et al. (2001) GLUT4 translocation precedes the stimulation of glucose uptake by insulin in muscle cells: potential activation of GLUT4 via p38 mitogen-activated protein kinase. Biochem J 359: 639-649

28. Sweeney G, Somwar R, Ramlal T, Volchuk A, Ueyama A, Klip A (1999) An inhibitor of p38 mitogen-activated protein kinase prevents insulin-stimulated glucose transport but not glucose transporter translocation in 3T3-L1 adipocytes and L6 myotubes. J Biol Chem 274:10071-10078

29. Konrad D, Somwar R, Sweeney G et al. (2001) The antihyperglycemic drug alpha-lipoic acid stimulates glucose uptake via both GLUT4 translocation and GLUT4 activation: potential role of $\mathrm{p} 38$ mitogen-activated protein kinase in GLUT4 activation. Diabetes 50:1464-1471

30. Standaert ML, Kanoh Y, Sajan MP, Bandyopadhyay G, Farese RV (2002) Cbl, IRS-1, and IRS-2 mediate effects of rosiglitazone on PI3 K, PKC-lambda, and glucose transport in 3T3/L1 adipocytes. Endocrinology 143:1705-1716

31. Kanoh Y, Bandyopadhyay G, Sajan MP, Standaert ML, Farese RV (2000) Thiazolidinedione treatment enhances insulin effects on protein kinase C-zeta /lambda activation and glucose transport in adipocytes of nondiabetic and Goto-Kakizaki type II diabetic rats. J Biol Chem 275: 16690-16696

32. Valverde AM, Teruel T, Navarro P, Benito M, Lorenzo M (1998) Tumor necrosis factor-alpha causes insulin receptor substrate-2-mediated insulin resistance and inhibits insulininduced adipogenesis in fetal brown adipocytes. Endocrinology 139:1229-1238

33. Vollenweider P, Menard B, Nicod P (2002) Insulin resistance, defective insulin receptor substrate 2-associated phosphatidylinositol-3' kinase activation, and impaired atypical protein kinase $\mathrm{C}$ (zeta/lambda) activation in myotubes from obese patients with impaired glucose tolerance. Diabetes 51:1052-1059

34. Tamori Y, Masugi J, Nishino N, Kasuga M (2002) Role of peroxisome proliferator-activated receptor-gamma in maintenance of the characteristics of mature 3T3-L1 adipocytes. Diabetes 51:2045-2055

35. Tennagels N, Telting D, Parvaresch S, Maassen JA, Klein HW (2001) Identification of Ser(1275) and Ser(1309) as autophosphorylation sites of the human insulin receptor in intact cells. Biochem Biophys Res Commun 282:387-393 
36. Dadke S, Kusari A, Kusari J (2001) Phosphorylation and activation of protein tyrosine phosphatase (PTP) 1B by insulin receptor. Mol Cell Biochem 221:147-154

37. Bandyopadhyay D, Kusari A, Kenner KA et al. (1997) Protein-tyrosine phosphatase $1 \mathrm{~B}$ complexes with the insulin receptor in vivo and is tyrosine-phosphorylated in the presence of insulin. J Biol Chem 272:1639-1645

38. Tao J, Malbon CC, Wang HY (2001) Insulin stimulates tyrosine phosphorylation and inactivation of protein-tyrosine phosphatase 1B in vivo. J Biol Chem 276:29520-29525

39. Mur C, Valverde AM, Kahn CR, Benito M (2002) Increased insulin sensitivity in IGF-I receptor-deficient brown adipocytes. Diabetes 51:743-754
40. Haj FG, Markova B, Klaman LD, Bohmer FD, Neel BG (2003) Regulation of receptor tyrosine kinase signaling by protein tyrosine phosphatase-1B. J Biol Chem 278:739744

41. Furnsinn C, Brunmair B, Meyer M et al. (1999) Chronic and acute effects of thiazolidinediones BM13.1258 and BM15.2054 on rat skeletal muscle glucose metabolism. Br J Pharmacol 128:1141-1148

42. Kumar N, Dey CS (2003) Development of insulin resistance and reversal by thiazolidinediones in $\mathrm{C} 2 \mathrm{C} 12$ skeletal muscle cells. Biochem Pharmacol 65:249-257 